\title{
Fabry disease in infancy and early childhood: a systematic literature review
}

\author{
Dawn A. Laney, MS', Dawn S. Peck, MS², Andrea M. Atherton, MS³, Linda P. Manwaring, MS, \\ Katherine M. Christensen, MS5, Suma P. Shankar, MD, PhD ${ }^{1}$, Dorothy K. Grange, MD ${ }^{4}$, \\ William R. Wilcox, MD, PhD ${ }^{1}$ and Robert J. Hopkin, MD ${ }^{6}$
}

Purpose: Fabry disease is a pan-ethnic, progressive, X-linked genetic disorder that commonly presents in childhood and is caused by deficient activity of the lysosomal enzyme alpha-galactosidaseA ( $\alpha$-gal A). Symptoms of Fabry disease in the pediatric population are well described for patients over five years of age; however, data are limited for infancy and early childhood. The purpose of this article is to delineate the age of detection for specific Fabry symptoms in early childhood.

Methods: A systematic retrospective analysis of PubMed indexed, peer-reviewed publications and case reports in the pediatric Fabry population was performed to review symptoms in patients reported before 5 years of age.

Results: The most frequently reported symptom in all age groups under 5 years was acroparesthesias/neuropathic pain, reported in
9 children, ranging in age from 2.0-4.0 years. Also notable is the frequency of gastrointestinal issues reported in 6 children aged 1.0-4.1 years of age.

Conclusion: This article finds clear evidence that symptoms can occur in early childhood, before age 5 years. Given early presenting symptoms and the ability to monitor these disease hallmarks, a timely referral to a medical geneticist or other specialty clinician experienced in managing children with Fabry disease is strongly indicated.

Genet Med advance online publication 18 September 2014

Key Words: Fabry disease; pediatric; children; newborn screening; symptoms

\section{INTRODUCTION}

Fabry disease (OMIM 301500) is a panethnic, X-linked, multisystemic, progressive lysosomal storage disorder caused by mutations in the GLA gene. These mutations result in decreased or deficient levels of the enzyme $\alpha$-galactosidase A ( $\alpha$-gal A) (EC 3.2.1.22). This $\alpha$-gal A deficiency causes the accumulation of globotriaosylceramide (GL3 or GB3) and related glycosphingolipids in many cell types, including, importantly, the vascular endothelium. ${ }^{1,2}$ Glycosphingolipid storage initiates a cascade of events, beginning with the dysfunction of basic metabolic processes on the cellular level and progressing to cell death, inflammatory events, and progressive major organ dysfunction. ${ }^{3}$ The incidence of Fabry disease ranges from 1 in 1,250-117,000 live male births worldwide, and an increased incidence of mutations are expected to result in later-onset Fabry symptoms. ${ }^{4-8}$ In the United States specifically, newborn screening has detected a high rate of Fabry disease among newborns, particularly those with GLA mutations currently categorized as later onset, with an incidence ranging from approximately 1 in 3,000 reported by the newborn screening data from Illinois and Missouri to 1 in 7,800 (males) in Washington State. ${ }^{9-12}$

Childhood symptoms of classic Fabry disease in children from 5 to 18 years old are increasingly well described. The largest review of pediatric patients with Fabry disease younger than 18 years included 352 patients ranging in age from 0 to 17 years, with a median age of 12 years. In the review of Fabry registry data by Hopkin et al., ${ }^{13}$ the median ages at symptom onset were 6 years in boys and 9 years in girls. The most frequently presenting symptom in boys and girls was neurologic pain, which was reported by $59 \%$ of boys (median age, 7 years) and $41 \%$ of girls (median age, 9 years). However, the age at symptom onset varied from individual to individual, even within the same family. ${ }^{13}$ Despite reports of burdensome symptoms in both boys and girls during childhood, Fabry disease is often considered an adult-onset disease by health-care providers who are less familiar with the condition. ${ }^{13,14}$ Although not typically life threatening, these symptoms clearly impact the health and function of affected children, both boys and girls..$^{13-16}$

The limited information available on Fabry disease in early childhood has made application of the guidelines of the pediatric Fabry Registry and American College of Medical Genetics and Genomics difficult in the population of children diagnosed prenatally or during infancy. ${ }^{17,18} \mathrm{~A}$ better understanding of the manifestations of Fabry disease and its effects during the newborn and early childhood period will provide valuable information on disease progression and assist clinicians in

\footnotetext{
${ }^{1}$ Division of Medical Genetics, Department of Human Genetics, Decatur, Georgia, USA; ${ }^{2}$ Division of Medical Genetics, University of Missouri Children's Hospital, University of Missouri Health System, Columbia, Missouri, USA; ${ }^{3}$ Section of Genetics, Children's Mercy Hospitals, Kansas City, Missouri, USA; ${ }^{4}$ Division of Genetics and Genomic Medicine, Department of Pediatrics, Washington University School of Medicine, St. Louis Children's Hospital, St. Louis, Missouri, USA; ${ }^{5}$ Division of Medical Genetics, SSM Cardinal Glennon Children's Medical Center, St. Louis, Missouri, USA; ' ${ }^{D}$ ivision of Human Genetics, Cincinnati Children’s Hospital Medical Center, Cincinnati, Missouri, USA. Correspondence: Dawn A. Laney (dawn.laney@emory.edu)
} 
personalizing published monitoring and management guidelines from birth. Herein we review published studies and case reports of the manifestations of Fabry disease in infants and children diagnosed during early childhood and discuss strategies and monitoring recommendations for newborns, infants, and young children with Fabry disease.

\section{MATERIALS AND METHODS}

In June 2014, we performed a systematic retrospective analysis of peer-reviewed publications and case reports about the pediatric Fabry population using PubMed. The initial PubMed search began with the search terms Fabry disease AND children. This search yielded a total of 504 references, of which 95 were relevant. Relevant papers had publication dates ranging from 1969 to 2014. Additional searches used terms that included Fabry disease AND pediatric, Fabry AND pediatric, and Fabry AND children. The number of relevant publications reviewed was expanded via references from available pediatric papers and other review papers. Only data that could be confirmed as data from patients in the age category of interest (before birth to younger than age 5 years) were included in this analysis. In total, a review of 120 papers provided information on 41 individual patients younger than 5 years of age, with 14 descriptive reports yielding specific case examples.

\section{RESULTS}

\section{Fabry disease in the fetus}

Prenatal and neonatal studies of the histopathology of Fabry disease before birth have confirmed that pathogenic GL3 accumulations occur in the maternal region of the placenta, fetal tissues, and the fetal placenta regions of affected males. ${ }^{19,20}$ In fetal males affected by Fabry disease, these accumulations have been found in renal, myenteric plexus, and liver cells. ${ }^{19-22}$ Corneal whorls also were detected as early as 22 weeks gestation in a male fetus. ${ }^{23}$ This prenatal storage suggests that the process of Fabry disease may lead to early childhood symptoms.

\section{Fabry disease during early childhood}

Table 1 summarizes the reports used for this review. The most frequently reported symptom in all age groups younger than 5 years was acroparesthesia/neuropathic pain, which was reported in 9 children ranging in age from 2.0 to 4.0 years. The frequency of gastrointestinal issues reported in six children aged 1.0-4.1 years is also notable. Table 2 lists classic Fabry disease symptoms and highlights the youngest age at which they have been reported in the literature.

The scarcity of published data on the impact and symptoms of Fabry disease in pediatric patients younger than 5 years of age is in part because of the small number of patients identified during early childhood and in part because of the limited assessments performed on and reported for patients in this age group. Before 2003, Fabry disease did not have a US Food and Drug Administration-approved treatment, women were mistakenly considered unlikely to present with symptoms, and Fabry disease was often viewed as a later-onset condition.
Combined with concerns over the impact of preexisting conditions on insurance, patient autonomy, and worries about the medicalization of childhood, at-risk family members were often not tested or evaluated until they developed Fabry-related symptoms or were older than 18 years. ${ }^{24,25}$ Accordingly, before newborn screening, published information on symptom onset during ages 0 to 4 years was obtained primarily from symptomatic children in the cohort of affected family members of probands.

When symptoms are reported in a general summary of early childhood features (younger than 5 years), they most frequently include gastrointestinal dysfunction, episodic pain crises, hypohidrosis, acroparesthesia, corneal whorls, retinal vascular tortuosity, mean height/weight <50th percentile (boys only), and abnormal heat and cold tolerance. ${ }^{13,15,16,26-32}$ (Table 1).

Notably, most children (boys and girls) younger than 5 years of age in the literature are reported to have only one or two of these most frequent symptoms. The variability in symptom onset is likely due to low numbers; a focus on a single-organ symptom assessment based on specialty; difficulties assessing pain in nonverbal infants and small children; differences in assessing pediatric symptoms (questions asked, patient/parent self-report, objective assessment); gender differences in symptom onset; and the variable nature of Fabry disease. Given the small number of cases reported in the literature and that a small number of both males and females in the $<5$-year-old population have symptoms reported, data are not analyzed separately by gender. The limited genotype data available for published cases also prevents meaningful analysis of genotype-phenotype correlations in this article. Each case with available demographics and genotype is listed in Table 1.

Heat/cold intolerance. Heat and cold intolerance in the youngest pediatric patients affected by Fabry disease is believed to result from abnormal autonomic nerve function and infiltration of the sweat glands by storage material, resulting in symptoms that include hypohidrosis, exercise intolerance, and pain. In the heat, affected patients may present with flushing, onset of neuropathic pain, headaches, fatigue, or heat stroke. In the cold, patients may complain of increased pain or numbness in the extremities. ${ }^{15,33,34}$ In the under 5-year-old age group, there are reports of three children affected by heat and cold intolerance at ages 3.5, 4.0, and 4.4 years. In all three of the reported cases, temperature sensitivity was associated with hypohidrosis or decreased sweating. Only one case of hypohidrosis without heat and cold intolerance at age 2.5 years was reported.

Neurological symptoms. The neurological symptoms of Fabry disease in the youngest pediatric population are related predominantly to a small-fiber neuropathy, leading to exercise intolerance, pain in the hands and feet, hypohidrosis, and periodic acute pain crises. Autonomic nervous system dysfunction may also be an accompanying feature of Fabry disease, causing gastrointestinal issues. ${ }^{35}$ Neuropathic pain (including chronic pain and acute pain crises) in the hands and 

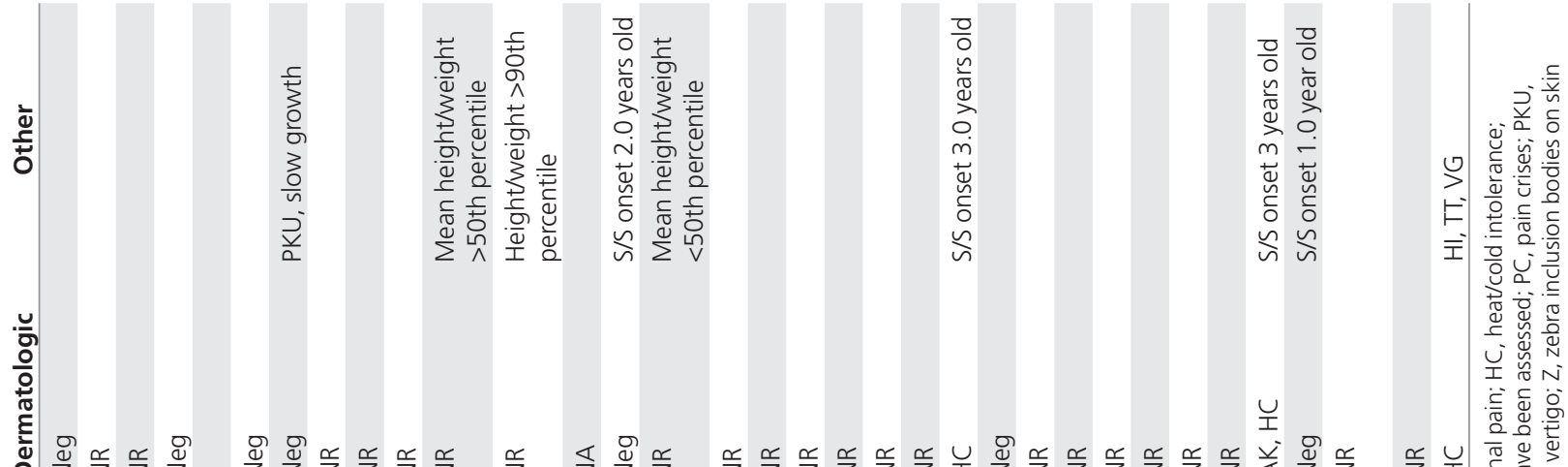

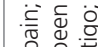

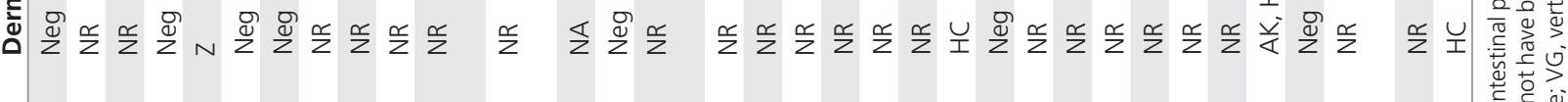

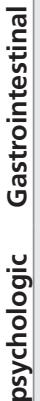

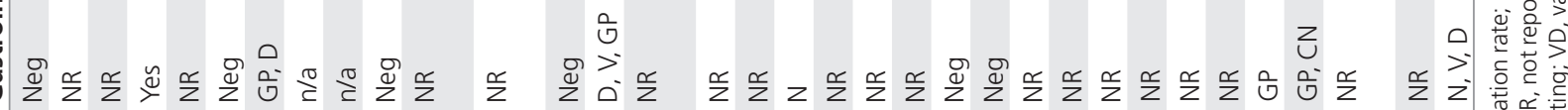
กิ. 产递

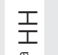

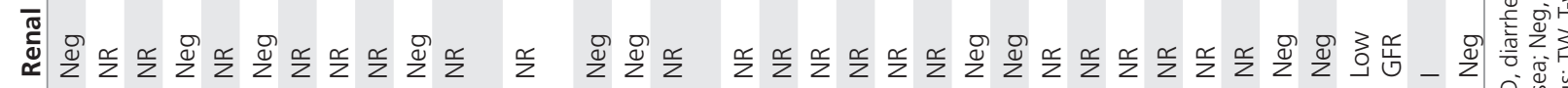

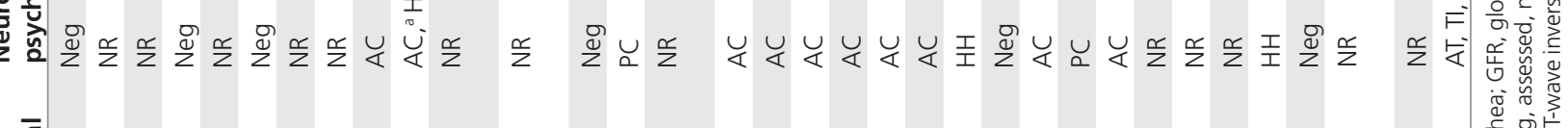

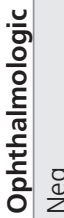
罂

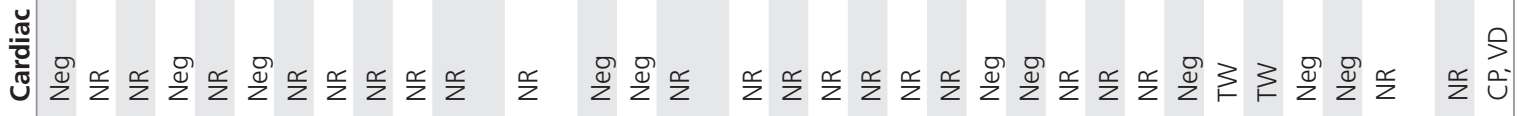

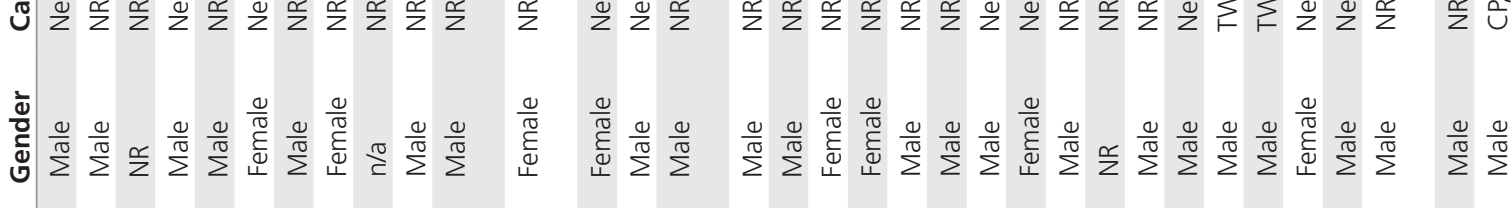
空

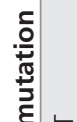

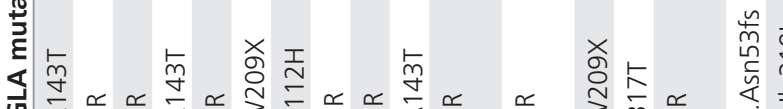

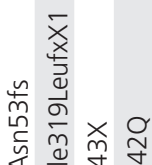

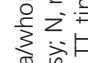
焉嗃 践 每 蛋 就离部 등 흥

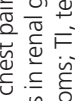

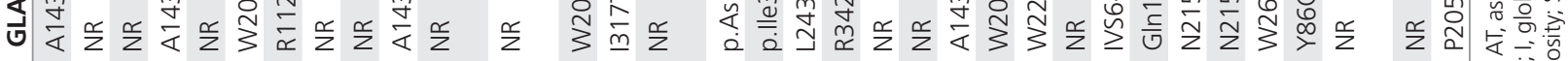

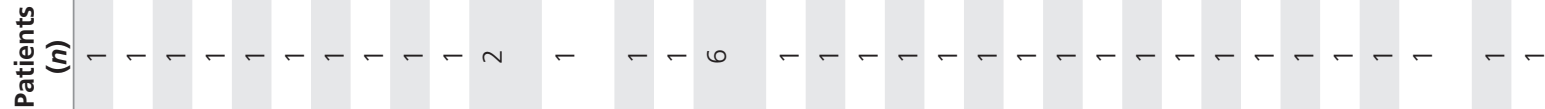
产 
Table 2 Summary of reported clinical manifestations in Fabry patients (newborn-4 years) 2, 13,16,27-32,36,40,41,51

\begin{tabular}{ll} 
Fabry-related signs and symptoms & \multicolumn{1}{|c}{$\begin{array}{c}\text { Earliest } \\
\text { report of } \\
\text { symptom }\end{array}$} \\
\hline $\begin{array}{l}\text { Storage of globotriaosylceramide found in organs on } \\
\text { biopsy }\end{array}$ & Prenatal \\
\hline $\begin{array}{l}\text { Corneal whorls/verticillata } \\
\text { Gastrointestinal problems, including nausea, vomiting, } \\
\text { diarrhea, constipation, and abdominal pain }\end{array}$ & $\begin{array}{l}\text { Prenatal/ } \\
\text { newborn }\end{array}$ \\
\hline Slow growth in boys (mean height/weight <50th percentile) & 1.0 year \\
\hline $\begin{array}{l}\text { Intermittent acroparesthesia/neuropathic pain triggered by } \\
\text { stress, heat, fatigue, or exercise }\end{array}$ & 2.0 years \\
\hline Hypohidrosis or anhidrosis & 2.5 years \\
\hline $\begin{array}{l}\text { Fabry crises of agonizing neuropathic pain typically begin in } \\
\text { the hands and feet and may radiate proximally }\end{array}$ & 2.5 years \\
\hline Heat, cold, and/or exercise intolerance & 3.5 years \\
\hline Retinal vascular tortuosity & 4.0 years \\
\hline Tinnitus/vertigo & 4.0 years \\
\hline Low glomerular filtration rate & 4.0 years \\
\hline $\begin{array}{l}\text { T-wave inversion on electrocardiogram } \\
\text { Trivial cardiac valve disease }\end{array}$ & 4.0 years \\
\hline Angiokeratoma & 4.0 years \\
\hline & 4.4 years \\
\hline
\end{tabular}

feet (acroparesthesia) and tingling are usually intermittent in this population and triggered by extreme temperatures, fevers, fatigue, stress, overheating, or exercise. ${ }^{34,36,37}$ There are published reports of 10 children in the early childhood age group with acroparesthesia/neuropathic pain, ranging in age from 2.0 to 4.0 years. There are also reports of pain crises specifically in one 2.5-year-old and one 4.0-year-old. . $^{13,16,27,28,31,38-41}$

Although not as common in the population younger than 5 years of age, children affected by Fabry disease can exhibit reduced sweating or hypohidrosis, as found and reported in four children ages 2.5-4.0 years. ${ }^{16,41}$ This may combine with autonomic dysfunction to result in exercise and heat intolerance, although it has not been studied in children younger than age $5.42,43$

Early microvascular cerebral involvement in the form of white matter lesions has been demonstrated by magnetic resonance imaging in asymptomatic patients as young as 8 years of age. We found no reports of brain magnetic resonance imaging in children younger than 5 years of age. ${ }^{44}$

Growth. Fabry disease is reported to affect the rate of physical growth and onset of puberty in boys. ${ }^{13}$ However, in the Fabry Registry, three male infants (ages 0 to $<2$ years) and six in early childhood (ages 2 to $<5$ years) were, on average, between the 40 th and 50th percentiles for height and weight. The only female infant between ages 0 and $<2$ was at the 90th percentile for height and weight. The average height and weight for pediatric patients aged 2 to 5 years differed by gender. The six described boys were between the 40th and 45th percentiles for height and weight, and the three girls were at the 50th percentile, with weight at the 70 th percentile. ${ }^{13}$ This supports the conclusion that growth problems are probably not part of infancy and are minimal during early childhood because there is limited disturbance of early growth. The growth abnormality seen in older children and teens likely results from progressive disease.

Dermatologic symptoms. Angiokeratomas are frequently found in patients with Fabry disease who are older than 5 years of age. In our review, however, only one patient (aged 4.0 years) was reported to exhibit visible angiokeratomas. ${ }^{41}$ During the literature review, there was also one case report of a 12-month-old boy found to have zebra inclusions in his endothelial and perithelial cells on skin biopsy despite the lack of visible angiokeratomas. ${ }^{45}$ In affected patients, changes in angiokeratomas do not usually correlate to disease severity; they may rarely occur in a friction-prone location, resulting in bleeding or discomfort for the patient. Recognizing and monitoring this Fabry disease feature may improve quality of life by identifying irritants and avoiding concern/misdiagnosis by general practitioners unfamiliar with this predominantly benign skin finding.

Ophthalmologic symptoms. Ophthalmologic findings (corneal whorls, vessel dilation, and tortuosity) may present in childhood before the presentation of other more severe symptoms. ${ }^{32}$ Fabry-related ophthalmologic manifestations in the early childhood population usually do not affect vision. In the early childhood age group, there are six symptomatic children reported: five with corneal whorls (0.5-4.1 years old) and a 4 -year-old with retinal vascular tortuosity. ${ }^{29,32,41}$ Reported data suggest that the presence of retinal vascular tortuosity can be correlated with disease severity in children. ${ }^{32}$

Audiologic symptoms. Audiologic symptoms in the pediatric population with Fabry disease are usually related to highfrequency hearing loss or intermittent tinnitus. ${ }^{46}$ Fabry-related hearing symptoms were reported in only one 4-year-old with auditory issues and included hearing impairment, tinnitus, and vertigo. ${ }^{32}$ A study of 14 audiograms in young children by Keilmann et al. ${ }^{46,47}$ found results consistent with reports that most children affected by Fabry disease have normal hearing.

Gastrointestinal symptoms. Gastrointestinal symptoms in the pediatric population with Fabry disease are often reported as abdominal pain, nausea, vomiting, bloating, and alternating episodes of diarrhea and constipation. These symptoms are probably related to gastrointestinal dysmotility caused by autonomic dysfunction. ${ }^{36,48}$ In early childhood reports, gastrointestinal issues were reported in six children 1.0-4.1 years of age. Specific issues included gastrointestinal pain in four children, as well as additional findings that included one or more of diarrhea, constipation, nausea, and vomiting in three children. . $^{32,41,49}$

Cardiac symptoms. Cardiac abnormalities, such as chest pain, minor cardiac valve disease, increased left ventricular mass 
(when indexed for body size), prolongation of the PR interval, and T-wave inversion indicating repolarization changes in lateral leads on electrocardiography have been detected in a few young pediatric patients affected by Fabry disease. ${ }^{16,30,50}$ In 2013 , a small-scale study by Havranek et al. ${ }^{30}$ of four early pediatric patients (under 5 years of age) who were naive to therapy found two 4-year-old boys exhibiting a T-wave inversion, indicating repolarization changes in lateral leads on electrocardiography. In addition, Allen et al. ${ }^{32}$ reported a 4 -year-old with chest pain and trivial valve disease, including tricuspid and mitral regurgitation. Life-threatening cardiac symptoms, such as severe arrhythmias and cardiac failure, are not reported in the pediatric population before the teenage years. ${ }^{30}$

Renal symptoms. Najafian et al. ${ }^{51}$ reported storage of GL3 in biopsied kidneys as early as age 4 years, with GL3 inclusions in all glomerular cell types. Reports of clinical renal involvement in the pediatric population with Fabry disease are limited but may include microalbuminuria, proteinuria, and reduced glomerular filtration rates. Among the published cases of patients younger than age 5 years, there is one reported case of clinical renal involvement in a 4 -year-old boy with an estimated glomerular filtration rate of $56 \mathrm{ml} / \mathrm{min} / 1.73$ $\mathrm{m}^{2}{ }^{13}$ Given the rarity of a significant decline in glomerular filtration rate or significant proteinuria in young children, its presence should prompt examination for other causes, including performing a renal biopsy. However, renal damage, including foot process effacement, occurs before there is clinically evident proteinuria. The small-scale study by Tøndel et al. ${ }^{52}$ reported that regularly monitored urine albuminto-creatinine ratios (ACRs), even within the normal range, can be informative about disease progression and treatment response. The study results indicated that when receiving enzyme replacement therapy (ERT), urine ACRs decrease in parallel to the decrease in podocyte GL3 deposits, even if the urine ACR was not above normal limits. ${ }^{52,53}$

\section{Monitoring suggestions}

The American College of Medical Genetics and Genomics Work Group on Diagnostic Confirmation of Lysosomal Storage Diseases, expert committees, and the Fabry Registry board have published guidelines and recommendations for the management and monitoring of pediatric and adult patients affected by Fabry disease. ${ }^{3,17,18,25,54}$ After confirming the diagnosis of Fabry disease, the American College of Medical Genetics and Genomics published guidelines for pediatric patients, which include the following:

- Referral to an appropriate medical professional, such as a medical geneticist and/or metabolic specialist, for a baseline evaluation of Fabry-related symptoms and a genetic counselor to discuss $\mathrm{X}$-linked inheritance pattern and recurrence risk

- Discussion of the natural history of Fabry disease

- Medical history review, with a focus on gastrointestinal symptoms, pain, sweating, and heat and cold intolerance
- Baseline physical exam and vital signs, including height, weight, and blood pressure

- Construction of a detailed family history to identify other at-risk family members

- Baseline diagnostic studies as appropriate for age group, which may include electrocardiogram, echocardiogram, ophthalmologic examination, renal function tests (glomerular filtration rate, microalbumin, albumin, and protein), and plasma and/or urine GL3

- Genetic counseling for family, including X-linked inheritance pattern, recurrence risk, and identification/testing of at-risk family members

- Monitoring every 6-12 months by a medical professional familiar with Fabry disease, such as a medical geneticist or metabolic specialist, to monitor patient for the onset of Fabry-related symptoms

- Development of a comprehensive monitoring and treatment plan, including the option of ERT

When utilizing these guidelines, attention to age-specific validity should be given to the infant to 4 -year-old age group in light of available published data on timing and severity of symptom onset. The core elements of a baseline assessment in this population should focus on referral to a medical professional experienced in the care and treatment of Fabry disease assessment of the child for pain (extremities and gastrointestinal), growth, heat/cold intolerance, and renal function (focused on routine urine ACR every 6-12 months); education of the parents about the identification and management of early symptoms of Fabry disease (heat/cold intolerance, gastrointestinal symptoms, and pain); genetic counseling about the natural history and inheritance of Fabry disease; construction of a detailed family history to identify and test at-risk family members; and creation of a detailed monitoring and treatment plan for the condition. The detailed monitoring and treatment plan should include a preliminary determination of visit frequency during the first few years of life. This plan should be considered flexible and be reassessed on a regular basis because some children may experience the onset of early gastrointestinal or pain symptoms that would require more intensive monitoring and treatment. However, given no evidence of clinically significant cardiac findings in patients younger than 4 years of age, baseline cardiology studies may be delayed until at least age 4 years.

Interpreting biomarkers in this population can be difficult. As Barr et al. ${ }^{55}$ found in their biomarker studies of newborn urine, the concentrations of GL3 in infants before 30 days of age are variable, unreliable, and may be high even in normal infants. Therefore, urine GL3 concentration should not be used as a prognostic biomarker before 30 days of age. ${ }^{55}$

Cases ascertained via newborn screening often benefit from genotype and family studies to clarify predicted disease severity. Although there can be intrafamilial variability of symptoms, much can still be gained by looking at other affected family members. In family members who have apparent end-organ damage related to Fabry disease, biopsies looking for GL3 
storage in affected organs can clarify the actual severity of Fabry disease. $^{56}$

Beyond diagnostic studies, monitoring neurological pain in very early childhood is difficult because of limited communication options with patients younger than 2 years of age. After 3-4 years of age, children are more verbal and better able to explain their pain, although it may be conveyed in nontraditional words and phrases such as "sharp sand in my shoes" or "ants biting me." Capturing information on episodic pain in children 2 months to 7 years of age could be attempted using validated early childhood surveys, such as the Face, Legs, Activity, Cry, Consolability behavioral pain scores ${ }^{57}$ or the Fabry-Specific Pediatric Health and Pain Questionnaire in older verbal children. ${ }^{58}$ In the meantime, each Fabry patient's pain should be monitored and treated on a case-by-case basis without ignoring possible symptoms of other common concomitant complaints, such as chronic otitis media unrelated to Fabry disease.

Given the possible bias toward classical symptom onset in published data and symptom variability, each patient's monitoring schedule should be personalized based on their genotype, symptoms, and additional family information. An experienced medical geneticist, metabolic specialist, or other specialty clinician experienced in managing children with Fabry disease will be best positioned to personalize monitoring guidelines using any available information on family members, patient assessment, and genotype.

\section{Treatment suggestions}

Intravenous ERT for Fabry disease with agalsidase- $\beta$ (Fabrazyme, Genzyme, Cambridge, MA) was approved in the United States in 2003 and is also currently available in many countries outside the United States. Another ERT used in the treatment of Fabry disease is agalsidase- $\alpha$ (Replagal, Shire Human Genetic Therapies, Lexington, MA). As of 2014, agalsidase- $\alpha$ is approved for the treatment of Fabry disease in Europe, Canada, and Australia but not in the United States.

Clinical trials have found ERT with agalsidase- $\alpha$ and agalsidase- $\beta$ to be effective in reducing plasma and tissue concentrations of GL3 in the vascular endothelium of the kidney, skin, and heart. Patients also report improvements in pain and gastrointestinal symptoms. Clinical trials have demonstrated the safety and effectiveness of both preparations of ERT. ${ }^{36,42,59-61}$

In theory, the earlier treatment with ERT starts, the greater the potential benefit for patients. This improved response with earlier treatment is supported by a small-scale study indicating that long-term ERT at a dosage of $1 \mathrm{mg} / \mathrm{kg}$ every 2 weeks begun in pediatric patients (four patients 7 to 18 years of age) can result in improved urinary ACR, substantial GL3 clearance of the mesangial and glomerular endothelial cells of the kidney, and dose-dependent clearance of renal podocyte inclusions. ${ }^{52,53}$

Several studies and case reports have provided subjective and objective support that ERT with agalsidase- $\alpha$ or agalsidase- $\beta$ is beneficial and safe in the pediatric population, with data on the earliest patient in published retrospective and prospective safety studies beginning at age 2.5 years. ${ }^{13,15,41,52,62-67}$ Patients report subjective improvements in pain, heat intolerance, gastrointestinal symptoms, and sweating. They also report significant reductions in school absences due to sickness. ${ }^{64}$ Objective measurements include decreases in plasma and dermal concentrations of GL3 and reductions in the use of anticonvulsants for neuropathic pain. . $^{13,41,63,64,67}$

However, long-term data regarding outcome with early treatment of young children or even adolescents are currently limited; the longest prospective studies follow patients for 5 years beginning at the earliest at age 7 years..$^{52}$ Given these limited data, it is difficult to draw true conclusions about the long-term impact of early treatment begun before 5 years of age. Clearly, the data are skewed to reflect those patients who have presented with symptoms, rather than reflecting a natural history of the disease in this young age group. In addition, in global practice, there is wide variability in the use of ERT, with some physicians beginning presymptomatic therapy at a young age and others waiting until end-organ damage is evident. ${ }^{17}$ This may in part be because of parents and practitioners who want to avoid the medicalization of childhood as well as avoid the cost and invasiveness of ERT. ${ }^{40}$

Newborn screening has opened up the possibility for very early treatment and optimal symptomatic management of young children affected by Fabry disease; however, today there are no clear guidelines regarding when to initiate treatment with ERT among the very young. . $^{13,68}$ The goal would be to initiate treatment to prevent the onset of symptoms while avoiding unnecessary medicalization of childhood. This optimal time point is elusive and not yet defined. The decision to initiate ERT should be made according to the clinical judgment of the managing metabolic physician in conjunction with the patient's family with reference to all available data on family history, genotype, and the patient's symptoms. This decision should be informed with the knowledge that GL3 accumulation is progressive, end-organ damage is largely irreversible, and there is an improved response in Fabry disease with early treatment. ${ }^{17,36,58,60}$

Having said this, ERT is not a "cure" for Fabry disease and does not eliminate the need for concomitant medications, precautions to avoid pain triggers, or ongoing monitoring., ${ }^{3,17}$ Nondrug therapies such as the identification and avoidance of conditions that increase pain or gastrointestinal issues, including extreme heat or cold or physical exertion, should be discussed with parents at the time of diagnosis. ${ }^{36}$ Although symptom-specific medications-such as carbamazepine to treat chronic acroparesthesia, angiotensin-converting enzyme inhibitors and angiotensin receptor blockers to reduce levels of proteinuria, and antidepressants-may not typically be needed in early childhood, health-care providers should discuss the use of these medications as needed. ${ }^{48}$ Beyond individual exercise and temperature intolerance, there are currently no specific dietary or activity restrictions reported or suggested for patients diagnosed with Fabry disease in early childhood.

Clinical research trials investigating other treatment options, including oral chaperone therapy, oral substrate reduction therapy, improved ERT agents, and gene therapy, are under way at various stages of progression. ${ }^{69}$ Although it is unlikely that early 
treatment studies will be open to pediatric patients, information about the investigation of new treatment options may be useful for families.

\section{Conclusions}

Fabry disease is a variable condition with an insidious progression of subtle changes that begin before birth. This review article strives to consolidate the currently available information on symptoms and symptom onset during the early childhood period, before age 5 years.

We find clear evidence that symptoms can occur during early childhood, with well-documented cases as early as $2-3$ years. The presenting symptoms to monitor during early childhood include pain, heat sensitivity, and gastrointestinal disease, especially recurrent abdominal pain and diarrhea. However, the scope of this review is limited by the very small number of published reports of this population, which could bias results, skewing them toward more severe patients, whose early diagnosis of Fabry disease may influence symptom detection.

Based on this information, timely referral to a medical geneticist or other specialty clinician experienced in managing children with Fabry disease is strongly indicated early in childhood. An experienced clinician will modify and manage the recommended pediatric schedule of monitoring designed to balance the importance of good clinical monitoring and treatment early enough to avoid end-organ damage, while also avoiding the unnecessary "medicalization" of childhood. To expand our knowledge of Fabry disease onset during early childhood, a large-scale prospective clinical study that monitors symptoms is critical to provide much-needed data in this population and to help clarify best clinical practices.

Although controversial, newborn screening for Fabry disease, where it is already implemented, may provide the opportunity to monitor and treat patients at an earlier age, without a diagnostic odyssey lasting many years. However, because of limitations in phenotype/genotype correlations, it may be difficult to predict disease severity in a child, especially with novel de novo missense mutations. Severity may be easier to predict in cases with affected family members, but such predictions can be limited by the variability that can occur within families.

\section{ACKNOWLEDGMENTS}

The authors recognize all of the hardworking medical professionals and scientists striving to improve medical knowledge and outcomes in Fabry disease as well as the courageous patients and families who allow us the privilege of working with them. We also thank Cheryl T. Strauss for editorial assistance.

\section{DISCLOSURE}

D.A.L. has received research grants and speaker honoraria from Genzyme, Synageva, Amicus Therapeutics, and Shire. She also is a member of the Board of Advisors of the Fabry Registry and is compensated for her service by Genzyme Corporation. D.S.P., A.M.A., L.P.M., D.K.G., and K.M.C. declare no conflict of interest. S.P.S. has served as a consultant to Genzyme, BioMarin Pharmaceuticals,
Amicus Therapeutics, and Shire. She has received grant support from Genzyme, Shire, and BioMarin Pharmaceuticals. W.R.W. has served as a consultant to Genzyme, BioMarin Pharmaceuticals, Amicus Therapeutics, and Shire and as a member of Genzyme's speakers' bureau. He has received educational grants and research grant support from Genzyme. He is also a member of the Board of Advisors of the Fabry Registry and is compensated for these services by Genzyme. R.J.H. is a member of the Board of Advisors of the Fabry Registry and is compensated for these services by Genzyme Corporation. He is also a paid consultant for Genzyme on matters relating to Fabry disease and has received speaking fees and research grants from Genzyme related to Fabry disease.

\section{REFERENCES}

1. Desnick RJ, Ioannou Y, Eng CM. Alpha-galactosidase A deficiency: Fabry disease. In: Scriver CR, et al, eds. The Metabolic and Molecular Bases of Inherited Disease, 8th edn. New York: McGraw-Hill, 2001:3733-3774.

2. Eng CM, Fletcher J, Wilcox WR, et al. Fabry disease: baseline medical characteristics of a cohort of 1765 males and females in the Fabry Registry. $J$ Inherit Metab Dis 2007;30:184-192.

3. Eng CM, Germain DP, Banikazemi M, et al. Fabry disease: guidelines for the evaluation and management of multi-organ system involvement. Genet Med 2006;8: 539-548.

4. Inoue T, Hattori K, Ihara K, Ishii A, Nakamura K, Hirose S. Newborn screening for Fabry disease in Japan: prevalence and genotypes of Fabry disease in a pilot study. J Hum Genet 2013;58:548-552.

5. Meikle PJ, Hopwood JJ, Clague AE, Carey WF. Prevalence of lysosomal storage disorders. JAMA 1999;281:249-254.

6. Zarate YA, Hopkin RJ. Fabry's disease. Lancet 2008;372:1427-1435.

7. Hwu WL, Chien YH, Lee NC, et al. Newborn screening for Fabry disease in Taiwan reveals a high incidence of the later-onset GLA mutation c.936+919G>A (IVS4+919G>A). Hum Mutat 2009;30:1397-1405.

8. Spada M, Pagliardini S, Yasuda M, et al. High incidence of later-onset fabry disease revealed by newborn screening. Am J Hum Genet 2006;79:31-40.

9. Lin HY, Chong KW, Hsu JH, et al. High incidence of the cardiac variant of Fabry disease revealed by newborn screening in the Taiwan Chinese population. Circ Cardiovasc Genet 2009;2:450-456.

10. Brower A, Hoffman AJ, Thompson BH, Watson MS. Utilization of the newborn screening translational research network to advance research and clinical applications. Mol Genet Metab 2011;102:272.

11. Scott CR, Elliott S, Buroker N, et al. Identification of infants at risk for developing Fabry, Pompe, or mucopolysaccharidosis-I from newborn blood spots by tandem mass spectrometry. J Pediatr 2013;163:498-503.

12. Hopkins P. Missouri's first year experience with full population pilot screening for Pompe, Gaucher, Fabry and Hurler disorders using digital microfluidics methodology. Poster presentation at the 2014 Lysosomal Disease Network WORLD Meeting, San Diego, CA. Mol Genet Metab 2014;111 (Abstract).

13. Hopkin RJ, Bissler J, Banikazemi M, et al. Characterization of Fabry disease in 352 pediatric patients in the Fabry Registry. Pediatr Res 2008;64: 550-555.

14. Ries M, Gupta S, Moore DF, et al. Pediatric Fabry disease. Pediatrics 2005;115:e344-e355.

15. Ramaswami U, Whybra C, Parini R, et al.; FOS European Investigators. Clinical manifestations of Fabry disease in children: data from the Fabry Outcome Survey. Acta Paediatr 2006;95:86-92.

16. Ries M, Ramaswami U, Parini R, et al. The early clinical phenotype of Fabry disease: a study on 35 European children and adolescents. Eur J Pediatr 2003;162:767-772.

17. Wang RY, Bodamer OA, Watson MS, Wilcox WR; ACMG Work Group on Diagnostic Confirmation of Lysosomal Storage Diseases. Lysosomal storage diseases: diagnostic confirmation and management of presymptomatic individuals. Genet Med 2011;13:457-484.

18. Fabry Registry pediatric minimum recommended schedule of assessment. 2010. http://www.fabrazyme.com/global/freg_schedule_of_assessments.pdf. Accessed 12 June 2014.

19. Elleder M, Poupětová H, Kozich V. Fetal pathology in Fabry's disease and mucopolysaccharidosis type I. Cesk Patol 1998;34:7-12. 
20. Thurberg BL, Politei JM. Histologic abnormalities of placental tissues in Fabry disease: a case report and review of the literature. Hum Pathol 2012;43:610614.

21. Desnick RJ, Raman MK, Bendel RP, Kersey J, Lee JC, Krivit W. Prenatal diagnosis of glycosphingolipidoses: Sandhoff's (SD) and Fabry's diseases (FD). J Pediatr 1973:83:149.

22. Vedder AC, Strijland A, vd Bergh Weerman MA, Florquin S, Aerts JM, Hollak CE. Manifestations of Fabry disease in placental tissue. J Inherit Metab Dis 2006;29:106-111.

23. Tsutsumi A, Uchida Y, Kanai T, Tsutsumi O, Satoh K, Sakamoto S. Corneal findings in a foetus with Fabry's disease. Acta Ophthalmol (Copenh) 1984:62:923-931.

24. American College of Medical Genetics Board of Directors. American Society of Human Genetics Board of Directors. Points to consider: ethical, legal and psychosocial implications of genetic testing in children and adolescents. Am J Hum Genet 1995; 57:1233-1241.

25. Bennett RL, Hart KA, O'Rourke $E$, et al. Fabry disease in genetic counseling practice: recommendations of the National Society of Genetic Counselors. J Genet Couns 2002;11:121-146

26. Ramaswami U, Wendt S, Pintos-Morell G, et al. Enzyme replacement therapy with agalsidase alfa in children with Fabry disease. Acta Paediatr 2007;96:122127.

27. MacDermot KD, Holmes A, Miners AH. Anderson-Fabry disease: clinical manifestations and impact of disease in a cohort of 60 obligate carrier females. J Med Genet 2001;38:769-775

28. MacDermot KD, Holmes A, Miners AH. Anderson-Fabry disease: clinical manifestations and impact of disease in a cohort of 98 hemizygous males. J Med Genet 2001;38:750-760.

29. Spaeth GL and Frost P. Fabry's disease. Its ocular manifestations. Arch Ophth 1969:74:760-769.

30. Havranek S, Linhart A, Urbanova Z, Ramaswami U. Early cardiac changes in children with anderson-fabry disease. JIMD Rep 2013;11:53-64.

31. Liu YP, Huang $Y$, Wang $Q$, et al. A boy with Fabry disease with the onset at the age of four. Beijing Da Xue Xue Bao 2013;45:307-311.

32. Allen LE, Cosgrave EM, Kersey JP, Ramaswami U. Fabry disease in children: correlation between ocular manifestations, genotype and systemic clinical severity. Br J Ophthalmol 2010;94:1602-1605.

33. Lidove O, Kaminsky P, Hachulla E, et al.; FIMeD investigators. Fabry disease 'The New Great Imposter': results of the French Observatoire in Internal Medicine Departments (FIMeD). Clin Genet 2012;81:571-577.

34. Hilz MJ, Stemper B, Kolodny EH. Lower limb cold exposure induces pain and prolonged small fiber dysfunction in Fabry patients. Pain 2000;84:361-365.

35. Geevasinga N, Tchan M, Sillence D, Vucic S. Upregulation of inward rectifying currents and Fabry disease neuropathy. J Peripher Nerv Syst 2012;17:399-406.

36. Desnick RJ, Brady RO. Fabry disease in childhood. J Pediatr 2004;144(suppl 5):S20-S26.

37. Pintos-Morell G, Beck M. Fabry disease in children and the effects of enzyme replacement treatment. Eur J Pediatr 2009;168:1355-1363.

38. Gahl WA, Adamson M, Kaiser-Kupfer I, et al. Biochemical phenotyping of a single sibship with both cystinosis and Fabry disease. J Inherit Metab Dis 1985;8:127-131.

39. Ries $\mathrm{M}$, Mengel $\mathrm{E}$, Kutschke $\mathrm{G}$, et al. Use of gabapentin to reduce chronic neuropathic pain in Fabry disease. J Inherit Metab Dis 2002;26:413-414.

40. Bouwman MG, de Ru MH, Linthorst GE, Hollak CE, Wijburg FA, van Zwieten MC. Fabry patients' experiences with the timing of diagnosis relevant for the discussion on newborn screening. Mol Genet Metab 2013;109:201-207.

41. Ramaswami U, Parini R, Kampmann C, Beck M. Safety of agalsidase alfa in patients with Fabry disease under 7 years. Acta Paediatr 2011;100:605-611.

42. Schiffmann R. Neuropathy and Fabry disease: pathogenesis and enzyme replacement therapy. Acta Neurol Belg 2006;106:61-65.

43. Sun A, Hernandez C, Wilcox W. Pulmonary disease and exercise intolerance in boys with Fabry disease: a pilot study. Poster Presentation at the 2012 Lysosomal Disease Network WORLD Meeting, San Diego, CA. Mol Genet Metab 2012 (Abstract).

44. Cabrera-Salazar MA, O'Rourke E, Charria-Ortiz G, Barranger JA. Radiological evidence of early cerebral microvascular disease in young children with Fabry disease. J Pediatr 2005;147:102-105.
45. Breathnach SM, Black MM, Wallace HJ. Anderson-Fabry disease. Characteristic ultrastructural features in cutaneous blood vessels in a 1-year-old boy. $\mathrm{Br} J$ Dermatol 1980;103:81-84.

46. Keilmann A. Inner ear function in children with Fabry disease. Acta Paediatr Supp/ 2003;92:31-2; discussion 27.

47. Keilmann A, Hajioff D, Ramaswami U; FOS Investigators. Ear symptoms in children with Fabry disease: data from the Fabry Outcome Survey. J Inherit Metab Dis 2009;32:739-744.

48. Burlina AP, Sims KB, Politei JM, et al. Early diagnosis of peripheral nervous system involvement in Fabry disease and treatment of neuropathic pain: the report of an expert panel. BMC Neurol 2011;11:61.

49. Concolino D, Rapsomaniki M, Disabella E, et al. Co-existence of Phenylketonuria and Fabry disease on a 3 year-old boy: case report. BMC Peds 2010;10:32-34.

50. Kampmann C, Wiethoff CM, Whybra C, Baehner FA, Mengel E, Beck M. Cardiac manifestations of Anderson-Fabry disease in children and adolescents. Acta Paediatr 2008;97:463-469.

51. Najafian B, Svarstad E, Bostad L, et al. Progressive podocyte injury and globotriaosylceramide (GL-3) accumulation in young patients with Fabry disease. Kidney Int 2011;79:663-670.

52. Tøndel C, Bostad L, Larsen KK, et al. Agalsidase benefits renal histology in young patients with Fabry disease. J Am Soc Nephrol 2013;24:137-148.

53. Kanai T, Yamagata T, Ito T, et al. Foot process effacement with normal urinalysis in classic fabry disease. JIMD Rep 2011;1:39-42.

54. Laney DA, Bennett RL, Clarke V, et al. Fabry disease practice guidelines: recommendations of the National Society of Genetic Counselors. J Genet Couns 2013;22:555-564.

55. Barr C, Clarke JTR, Ntwari A, Drouin R, Auray-Blais C. Fabry disease urinary globotriaosylceramide/creatinine biomarker evaluation by liquid chromatography-tandem mass spectrometry in healthy infants from birth to 6 months. Mol Genet Metab 2009;97:278-283.

56. Terryn W, Vanholder R, Hemelsoet D, et al. Questioning the pathogenic role of the GLA p.Ala143Thr "Mutation" in Fabry disease: implications for screening studies and ERT. JIMD Rep 2013;8:101-108.

57. Merkel S, Voepel-Lewis T, Malviya S. Pain assessment in infants and young children: the FLACC scale. Am J Nurs 2002;102:55-58.

58. Ramaswami U, Stull DE, Parini R, et al.; FOS Investigators. Measuring patient experiences in Fabry disease: validation of the Fabry-specific Pediatric Health and Pain Questionnaire (FPHPQ). Health Qual Life Outcomes 2012; 10:116.

59. Banikazemi M, Bultas J, Waldek S, et al.; Fabry Disease Clinical Trial Study Group. Agalsidase-beta therapy for advanced Fabry disease: a randomized trial. Ann Intern Med 2007;146:77-86.

60. Desnick RJ, Brady R, Barranger J, et al. Fabry disease, an under-recognized multisystemic disorder: expert recommendations for diagnosis, management, and enzyme replacement therapy. Ann Intern Med 2003;138:338-346.

61. Schiffmann R. Natural history of Fabry disease in males: preliminary observations. J Inherit Metab Dis 2001;24:15-17.

62. Ries M, Clarke JT, Whybra C, et al. Enzyme replacement in Fabry disease: pharmacokinetics and pharmacodynamics of agalsidase alpha in children and adolescents. J Clin Pharmacol 2007;47:1222-1230.

63. Ries M, Clarke JT, Whybra C, et al. Enzyme-replacement therapy with agalsidase alfa in children with Fabry disease. Pediatrics 2006;118:924-932.

64. Wraith JE, Tylki-Szymanska A, Guffon N, et al. Safety and efficacy of enzyme replacement therapy with agalsidase beta: an international, open-label study in pediatric patients with Fabry disease. J Pediatr 2008;152:563-70, 570.e1.

65. Martin-Suárez I, Suárez-Marrero C. Beneficial effects of long-term enzyme replacement therapy in a child with Fabry disease. Int I Clin Pract 2010;64:995.

66. Germain DP, Waldek S, Banikazemi M, et al. Sustained, long-term renal stabilization after 54 months of agalsidase beta therapy in patients with Fabry disease. J Am Soc Nephrol 2007;18:1547-1557.

67. Ramaswami U. Fabry disease during childhood: clinical manifestations and treatment with agalsidase alfa. Acta Paediatr Supp/ 2008;97:38-40.

68. Najafian B, Mauer M, Hopkin RJ, Svarstad E. Renal complications of Fabry disease in children. Pediatr Nephrol 2013;28:679-687.

69. Motabar O, Sidransky E, Goldin E, Zheng W. Fabry disease - current treatment and new drug development. Curr Chem Genomics 2010;4:50-56. 